\title{
Exploration and Progress of Mobile Medical Treatment in Our Hospital
}

\author{
Hongcheng Tian ${ }^{1}$, Cheng Dong ${ }^{2}$, Taihuan $\mathrm{Li}^{1}$, and Jinkui $\mathrm{Ma}^{3+}$ \\ ${ }^{1}$ Information Department, The 309th Hospital of PLA, China \\ ${ }^{2}$ The Department of Medical Administration, The 309th Hospital of PLA, China \\ ${ }^{3}$ Health Care Office, The 309th Hospital of PLA, China
}

\begin{abstract}
With the development of mobile digital technologies and medical science, more and more people pay attention to mobile medical treatment in the circle of wisdom medical. This paper describes the definition, contents, architecture and three typical applications of mobile medical treatment, and introduces the exploration and progress of mobile medical treatment of our hospital in two aspects: the mobile digital hospital in the Internet and the mobile medical platform in the intranet of our hospital. Additionally, the nextstep schematizations in our hospital are presented, such as the mobile medical vehicles and the vehicle head magnetic resonance system. This paper is a valuable reference for researchers and engineers to carry out the further study on the mobile medical treatment.
\end{abstract}

Keywords: mobile medical treatment, exploration, progress

\section{Introduction}

In today china, the national economy is developing rapidly, the trend of aging is accelerating, the ecological environment is deteriorating, and peoples are falling over themselves for healthful lives and are making higher requests for medical services. However, perfect healthcare resource is centered in large medical centers and central cities due to uneven insufficient medical services and rareness of medical specialists in ordinary hospitals and cities. And a large number of people swarm into central hospitals. Thus, the problem, that medical treatment is difficult and expensive, is becoming increasingly prominent. This situation is aggregating the conflicts between doctors and patients. At present, the gap between peoples' requirement for health and fairness (and accessibility) of medical service is very wide.

The medical service is peoples' livelihood, and the 18th and 19th National Congresses of the Communist Party of China have made important instructions to improve national medical services. Especially, the report of the 19th party congress make clear requirement that a well-off society is built in an all-round way in 2020, everybody has access to basic medical service, and the government improves health level of all the people [1].

The Central Committee of the Communist Party of China puts forward the following pressing problems to the medical circle: how to excavate and take full advantage of the public medical service of whole society, how to reduce the cost of medical service and improve the level of medical service, and how to implement the strategic objective that every people has access to the basic medical service.

Hospitals are going into the information stage with the development of information technologies all over the world. The special advantages of mobile communication technology (networking, intellectualization, automatization, etc.) are applied to the circles of medical treatment and healthcare. Thus, mobile medical service becomes an important part of the development of strategic industry (Internet of Things).

\footnotetext{
${ }^{+}$Corresponding author. Tel.: +86 18612372885; fax: +86 01066711281.
}

E-mail address: majk00@163.com. 




Fig. 1: Architecture of mobile medical treatment.

This paper presents the definition, contents, architecture and three typical applications of mobile medical treatment. And this paper introduces the exploration and advances of mobile medical treatment of our hospital in two aspects: the mobile digital hospital in the Internet and the mobile medical platform in the intranet of our hospital (the intranet in our hospital is physically isolated from Internet). In addition, our hospital plans to carry out two projects: the mobile medical vehicles and the vehicle head magnetic resonance system.

The rest of the paper is organized as follows: In Section 2, we describe the definition, contents, architecture and three typical applications of mobile medical treatment. Section 3 introduces exploration and progress of mobile medical treatment in our hospital. In Section 4, we presents the next-step projects of our hospital in the mobile medical treatment. And in Section 5, the conclusions are given.

\section{Mobile Medical Treatment and Its Architecture}

According to the definition of Healthcare Information and Management Systems Society (HIMSS), Mobile medical treatment is to supply medical services and information through mobile communication technologies [2].

Mobile medical treatment includes (but are not limited to) the following aspects: appointment, remote consultation, electronic health records, medication management, chronic disease management, post acute care services, health inquiry, health evaluation, mobile doctor workstation, mobile nurse workstation, etc. [36].

Mobile medical treatment comprises three layers(Fig. 1): sensor layer, interconnecting layer and application layer [3-6].

Sensor layer consists of different mobile medical facilities. Interconnecting layer includes a series of mobile communication technologies, for example, dial network, digital subscriber line, Bluetooth, 3G(3rdgeneration), $4 \mathrm{G}$ (4th-generation), $5 \mathrm{G}$ (5th-generation), Wi-Fi and so on, providing the service of information exchange. Information that mobile medical facilities collect is sent to application layer through interconnecting layer. Application layer comprises typical mobile medical applications which include business process logics and backstage databases to implement wisdom medical.

\subsection{Mobile Medical Facilities}

Now, there are many mobile medical facilities, such as a pulse oximeter, a blood glucose meter, an electronic sphygmomanometer, a mobile ECG (electrocardiogram) monitoring terminal, a mobile ultrasound system, etc. 




Fig. 2: A 4G ambulance.

The mobile ultrasound system is a type of mobile medical facilities, including a portable ultrasonic detector and a mobile phone. The portable ultrasonic detector connects with the mobile phone. Ultrasonic images are displayed on the mobile phone and are sent to a long-distance terminal.

There are many other mobile medical facilities, such as an intelligent health integral machine, a home health instrument, a portable comprehensive detector, a teleconsultation instrument, etc. The intelligent health integral machine is a widely-used healthcare management system and it can be used to conduct the following inspection items: blood pressure, blood sugar, blood oxygen, body weight, body mass index (BMI), electrocardiogram (single lead electrocardiogram, multiple lead electrocardiogram), and body composition analysis (fat content, total moisture, muscle amount, percentage of body fat, body temperature, lung function, sports consumption, etc.)

\subsection{Mobile Communication Technologies}

Mobile communication technologies in the circle of mobile medical treatment mainly include the following technologies: Bluetooth, 2G, 3G, 4G, 5G, Wi-Fi and so on.

With the development of communication technologies, more advanced mobile communication technologies will provide faster and more stable interconnection services.

\subsection{Mobile Medical Applications}

There are many application cases in the circle of mobile medical treatment. Here, we only introduce three typical application systems: a neonatal management, a remote electrocardiogram(ECG) monitoring and a 4G ambulance (Fig. 2).

1) A Neonatal Management [7]

When a pregnant woman is in a hospital, a registration staff makes a wrist strap for the woman and writes her important information into the wrist strap through a RFID reader-writer. The nurse ties the wrist strap to the woman's wrist as her identity. The nurse conducts all the nursing services and updates corresponding electronic records inside the wrist strap whenever possible. After the infant is born, the nurse makes a wrist strap which matches the mother's wrist strap for the infant, forming a holonomic RFID record. When the infant comes to the mother, the nurse uses the portal RFID reader-writer to read the wrist straps of mother and infant in order to identify whether the infant matches the mother or not. When the infant departs the pediatric ward, the entrance guard system gives out the alarm.

2) A Remote ECG Monitoring [8]

Chinese remote ECG monitor is put into operation in Beijing, Xiamen, Xi'an and so on. The core facility is an individual ECG monitor whose function is to monitor the state of the carrier's heart whenever and wherever possible. After wearing the individual ECG monitor on the chest with the help of a doctor, the patient may go home. When the patient feels discomfortable, he may monitor his heart with the individual ECG monitor. The patient may send the collected information to the remote ECG monitoring center through GPRS or CD-MA1X. In the remote ECG monitoring center, cardiovascular specialists or physicians analyze 
and diagnose the ECG data from the patient, and response management advice to the patient through short messages.

3) 4G Ambulance [9]

Hangzhou urgent care centre cooperates with Zhejiang Mobile to pilot the $4 \mathrm{G}$ wisdom medical project. In detail, a $4 \mathrm{G}$ ambulance is equipped with a mobile workstation, and the ambulance may communicate with the urgent care centre through $4 \mathrm{G}$ network, an emergency physician in the ambulance may send the emergency information (vital sign or video of a patient) back to the urgent care center. Thus, the urgent care center may know the status of the patient and prepare to receive the patient before the patient arrives. If an emergency situation occurs, the emergency physician may communicate with the emergency experts in the urgent care center through the video or audio on the way to the urgent care center, and the emergency physician may rescue the patient under emergency experts' guidance.

\section{Exploration and Progress of Mobile Medical Treatment in Our Hospital}

Our hospital has constructed the prototype of mobile digital hospital in the Internet and inner mobile platform in the intranet of our hospital.

The target of mobile digital hospital is for patients to inquire the information of hospitals and experts, and to implement appointment, teleconsultation, health management, health education, and experience exchange among patients.

The target of inner mobile platform in our hospital is to implement mobile doctor workstation, mobile nurse workstation, remote visiting service, consultation management, medication management and so on.

\subsection{Mobile Digital Hospital}

Hospitals all over the country may join the platform of the mobile digital hospital. A doctor, who is registered in the platform, may set consulting time range on line and appointment number through his mobile phone or iPad; patients may inquire information of hospitals and experts, appoint, and upload his medical records and inspection reports in advance. The platform may extract the medical information or inspection reports of patients through secure gateway (or work done by hand) from joined hospitals. When the time comes, the doctor may make a diagnosis and give treatment to the patient through his mobile phone or iPad.

Patients may send their vital signs to doctors through wearable intelligent terminals for remote consultation, chronic disease management, health management and so on.

\subsection{Inner Mobile Platform in our Hospital}

Our hospital cooperates with China Mobile Communication Inc. and medical software companies to construct a wireless network covering the whole hospital on which a mobile medical application is based. This system integrates the quality management, and reconstructs the medical processes.

In this system, the preoperative communication between a doctor and a patient may be made before the sickbed. The doctor may get inspection results through iPad whenever possible and modify the operation scheme. A nurse may use the mobile nursing workstation through iPad to perform work exchange, three checks(check before the operation, check when operating, and check after the operation) and seven verifications(verify the bed number, verify the name, verify the drug, verify the dosage, verify the time, verify the potency, and verify the method) in order to reconstruct the data chains generated from the patients, nurses and drugs. According to the analysis of Motorola Inc., if a nurse's working time per day is 10 hours, she needs to walk 5 kilometers, but when she uses mobile nursing workstation, her walk distance can reduce to 3 kilometers. That is to say, the nurse can have more time to look after patients.

\section{The Next-Step Planning of Our Hospital in the Mobile Medical Treatment}

Our hospital plans to construct the following two systems: mobile medical vehicles and the vehicle head magnetic resonance system.

\subsection{Mobile Medical Vehicles}


The system consists of a remote command vehicle, a diagnostic and therapeutic vehicle, a medical insurance vehicle and an auxiliary inspection vehicle.

The remote command vehicle plays a role of organization and command. It is equipped with the vehicle satellite positioning and a 4G wireless network, and it can receive and send faxes and emails.

The diagnostic and therapeutic vehicle, equipped with infusion, oxygen uptaking, etc., may supply specialist services, covering internal medicine, forensic sursery, orthopaedics, dermatology, psychology and so on.

The medical insurance vehicle provides guarantees for the medical diagnostic, including several serials of insurance sets and systems, such as a vehicle operating room.

The auxiliary inspection vehicle can conduct a series of one-stop inspection services, such as a type-B ultrasonic, an electrocardiogram, a nuclear magnetic resonance, digital radiography, laboratory tests and so on.

\subsection{The Vehicle Head Magnetic Resonance System}

Cerebral apoplexy is the primary disease with high morbidity, disability and death rates in China. More than $90 \%$ of the patients with ischemic cerebral apoplexy fail to get timely thrombolytic therapy, because they are unable to reach hospitals timely. The pre-hospital delay is the major cause that misses the window time of thrombolytic therapy, and lack of understanding the symptoms of cerebral apoplexy, the masses cannot cognize the occurrence of cerebral apoplexy in time.

Focusing on this problem, there are three resolution strategies: the medical imaging diagnosis on cerebral apoplexy, the antedisplacement of thrombolytic therapy, and the mobile solution of the pre-hospital treatment of cerebral apoplexy.

The study on the vehicle head magnetic resonance system includes the four topics as below: (1) to develop the vehicle cranial magnetic resonance technologies; (2) to study the pre-hospital cerebral apoplexy treatment solutions, the correlative systems and products; (3) to integrate the pre-hospital mobile treatment platforms on cerebral apoplexy based on magnetic resonance; (4) to comprehensively evaluate pre-hospital treatment solutions of cerebral apoplexy. And the research target is to construct the mobile sustainable service model of the pre-hospital treatment on cerebral apoplexy. The four main research contents are described as follows:

The first is the key technology research and product development of the vehicle head magnetic resonance system. This part focuses on the design and optimization of large aperture and short magnets, RF coil technology, fast scanning sequence, picture archiving and communication systems (PACS), damping of magnet and system components, and layout optimization and integration among devices.

The second is the research and development of the mobile service integrated platform of the pre-hospital treatment on cerebral apoplexy. It focuses on the selection of the vehicle platforms, integration of mobile medical insurance equipments, mobile platform support system, vehicle control and scheduling system, and vehicle information technologies.

The third is the study of the clinical solution for the mobile pre-hospital cerebral apoplexy treatment. It focuses on organization structure, the responsibilities of each organization unit, the first-aid procedure, qualification requirements for organization units, and various specifications for the hierarchy of the mobile pre-hospital cerebral apoplexy treatment.

The fourth is the supporting system and comprehensive evaluation study on the solution of the mobile pre-hospital cerebral apoplexy treatment. It focus on the researches of technical system, policy system and comprehensive evaluation system.

\section{Conclusions}

This paper describes what mobile medical treatment is, and introduces the exploration and advances of mobile medical treatment of our hospital in two aspects: the mobile digital hospital and the mobile medical platform. In addition, two plans in our hospital are presented, such as the mobile medical vehicles and the 
vehicle head magnetic resonance system. In the next step, our hospital will improve and perfect the application of mobile medical treatment in order to supply convenient speedy medical services to patients.

\section{Acknowledgements}

This work is supported by China Postdoctoral Science Foundation under Grant 2015M572723 and the Key Hospital-level Project of the 309th Hospital of PLA (2017ZD-006).

\section{References}

[1] Xi J.P.. Xi Jinping's report at 19th Party Congress. http://cpc.people.com.cn/n1/2017/1028/c64094-29613660.html. 2017 (in chinese).

[2] Baidu. Mobile Health. http://baike.baidu.com/view/5373330.htm?fr=aladdin. 2014 (in chinese).

[3] Sultz H.A.. Health Care USA: Understanding Its Organization and Delivery. Jones \& Bartlett: Jones \& Bartlett Learning, 2016.

[4] Istepanian R.. M-Health: Emerging Mobile Health Systems. Springer: Springer, 2010.

[5] Istepanian R.. M-Health: Fundamentals and Applications. Wiley-IEEE: Wiley-IEEE Press, 2014.

[6] Mavhemwa P.M.. Effectiveness of an integrated m-health platform in malaria control. LAP LAMBERT: LAP LAMBERT Academic Publishing, 2012.

[7] Wang K.. Infant Management System Based on RFID and Internet Technologies. 1st International Conference on Information Science and Engineering (ICISE). 2009, pp. 3665-3668.

[8] Union of Remote ECG Monitoring. Description of Remote ECG Monitoring. http://ycxd.999120.net/ycjhjs.html. 2018 (in chinese).

[9] ZheJiang on line. Mobile 4G Ambulance. http://zjnews.zjol.com.cn/05-zjnews/system/2013/05/23/019357061.shtml. 2013(in chinese). 